\title{
Periconception glycaemic control in women with type 1 diabetes and risk of major birth defects: population based cohort study in Sweden
}

\author{
Jonas F Ludvigsson, ${ }^{1,2,3,4}$ Martin Neovius, ${ }^{5}$ Jonas Söderling, ${ }^{5}$ Soffia Gudbjörnsdottir, ${ }^{6,7}$ \\ Ann-Marie Svensson, ${ }^{6}$ Stefan Franzén,, ${ }^{6}$ Olof Stephansson, ${ }^{5,8}$ Björn Pasternak ${ }^{5,9}$
}

For numbered affiliations see end of article.

Correspondence to:

JF Ludvigsson Department

of Medical Epidemiology

and Biostatistics, Karolinska Institutet, 17177 Stockholm,

Sweden jonasludvigsson@

yahoo.com (or @ludvigsson on

Twitter)

Additional material is published online only. To view please visit the journal online.

Cite this as: $B M J$ 2018;362:k2638 http://dx.doi.org/10.1136/bmj.k2638

Accepted: 10 May 2018

\section{ABSTRACT}

OBJECTIVE

To examine the association between maternal type 1 diabetes and the risk of major birth defects according to levels of glycated haemoglobin ( $\mathrm{HbA} 1 \mathrm{C})$ within three months before or after estimated conception. DESIGN

Population based historical cohort study using nationwide health registers.

\section{SETTING}

Sweden, 2003-15.

\section{PARTICIPANTS}

2458 singleton liveborn infants of mothers with type 1 diabetes and a glycated haemoglobin measurement within three months before or after estimated conception and 1159865 infants of mothers without diabetes.

\section{MAIN OUTCOME MEASURES}

Major cardiac and non-cardiac birth defects according to glycated haemoglobin levels.

\section{RESULTS}

122 cases of major cardiac defects were observed among 2458 infants of mothers with type 1 diabetes. Compared with 15 cases of major cardiac defects per 1000 infants of mothers without diabetes, the rates among infants of mothers with type 1 diabetes were 33 per 1000 for a glycated haemoglobin level of $<6.5 \%$ (adjusted risk ratio $2.17,95 \%$ confidence interval 1.37 to 3.42 ), 49 per 1000 for $6.5 \%$ to $<7.8 \%$ (3.17, 2.45 to 4.11$), 44$ per 1000 for $7.8 \%$ to $<9.1 \%(2.79$, 1.90 to 4.12$)$, and 101 per 1000 for $\geq 9.1 \%(6.23,4.32$

\section{WHAT IS ALREADY KNOWN ON THIS TOPIC}

Type 1 diabetes in pregnant women has been linked to an increased risk of birth defects in offspring

Little data exist, however, on the role of glycaemic control

It is unclear if women meeting target levels recommended by guidelines ( $66.5 \%)$ are at increased risk of having offspring with birth defects

\section{WHAT THIS STUDY ADDS}

Among liveborn infants of mothers with type 1 diabetes, increasingly worse glycaemic control within three months before or after estimated conception was associated with a progressively increased risk of major cardiac defects

Even with glycated haemoglobin within recommended target levels ( $16.5 \%)$, the risk of major cardiac defects was increased more than twofold

The risk of major non-cardiac defects was not statistically significantly increased at any of four levels of glycated haemoglobin examined, although the study had limited statistical power for this outcome and was based on live births only to 9.00). The corresponding adjusted risk differences were 17 ( 5 to 36 ), 32 ( 21 to 46 ), 26 (13 to 46), and 77 (49 to 118) cases of major cardiac defects per 1000 infants, respectively. 50 cases of major non-cardiac defects were observed among infants of mothers with type 1 diabetes. Compared with 18 cases of major non-cardiac defects per 1000 infants of mothers without diabetes, the rates among infants of mothers with type 1 diabetes were 22 per 1000 for a glycated haemoglobin level of $<6.5 \%$ (adjusted risk ratio 1.18 , 0.68 to 2.07 ), 19 per 1000 for $6.5 \%$ to $<7.8 \%$ (1.01, 0.66 to 1.54$), 17$ per 1000 for $7.8 \%$ to $<9.1 \%(0.89$, 0.46 to 1.69$)$, and 32 per 1000 for $\geq 9.1 \%(1.68,0.85$ to 3.33$)$.

\section{CONCLUSIONS}

Among liveborn infants of mothers with type 1 diabetes, increasingly worse glycaemic control in the three months before or after estimated conception was associated with a progressively increased risk of major cardiac defects. Even with glycated haemoglobin within target levels recommended by guidelines ( $16.5 \%)$, the risk of major cardiac defects was increased more than twofold. The risk of major non-cardiac defects was not statistically significantly increased at any of the four glycated haemoglobin levels examined; the study had limited statistical power for this outcome and was based on live births only.

\section{Introduction}

Poor glycaemic control has been linked to both immediate (eg, ketoacidosis ${ }^{1}$ ) and long term (eg, mortality and cardiovascular disease ${ }^{23}$ ) complications in people with type 1 diabetes. The risk of pregnancy related complications is of particular relevance to women with type 1 diabetes. ${ }^{4}$ One of the most severe adverse pregnancy outcomes is birth defects, which may occur at a rate 2-4 times higher in the infants of mothers with type 1 diabetes than in infants of mothers without diabetes. $^{56}$

Data are limited on the association between glycaemic control measured through glycated haemoglobin (HbA1C) levels in pregnant women and birth defects in the offspring. Although most, ${ }^{4-13}$ but not all, ${ }^{14}$ studies indicate that glycated haemoglobin levels during pregnancy are associated with a risk of birth defects, earlier research had limited statistical power and did not differentiate between cardiac and non-cardiac defects. Furthermore, although guidelines from the American Diabetes Association and other major diabetes organisations recommend a target glycated haemoglobin of $<6.5 \%(<48 \mathrm{mmol} / \mathrm{mol})$ in 
early pregnancy, ${ }^{15-17}$ it is unclear whether there is no increase in risk of birth defects below this level and how the risk varies with increasing levels. A recent study from England and Wales reported that only one in six pregnant women with type 1 diabetes had a glycated haemoglobin of $<6.5 \%$ around the time of conception. $^{18}$

In this cohort study, we linked data from the Swedish national diabetes register with data from 1162323 pregnancies to investigate the association between type 1 diabetes and risk of major birth defects according to levels of glycated haemoglobin within three months before or after estimated conception.

\section{Methods}

Data sources

Each resident in Sweden is assigned a unique identifier, the personal identity number. ${ }^{19}$ Through the identifier we linked data on diabetes from the Swedish national diabetes register ${ }^{20}$ with the Swedish medical birth register, ${ }^{21}$ national patient register, ${ }^{22}$ cause of death register, ${ }^{23}$ total population register, ${ }^{24}$ and Swedish education register (see supplementary eMethods for further details). The data used were collected in routine healthcare and not specifically for this study.

\section{Participants}

Through the medical birth register, we identified singleton live births in Sweden with a delivery date between 1 January 2003 and 31 December 2014. We excluded births with missing maternal or infant personal identity numbers, missing or implausible gestational age ( $\leq 22$ weeks or $>44$ weeks), mothers aged less than 18 years (not covered by the national diabetes register), and mothers who did not live in Sweden during the year before conception. Furthermore, we excluded infants with chromosomal disorders (eg, Down's syndrome), genetic defects (eg, DiGeorge syndrome), birth defects of known causes (eg, dysmorphism due to warfarin), and viral infections associated with birth defects (eg, congenital rubella syndrome), to exclude cases not likely explained by diabetes (eTable 1). Using the national diabetes register, we identified mothers with a diagnosis of type 1 diabetes and a first registration any time before pregnancy and up to 91 days after conception, and who had one registered glycated haemoglobin value or more in the preconception period, defined as any time between 90 days before to 91 days after estimated conception (182 days in total). If a mother had two or more values in this time window, we used the last recorded measurement. We categorised glycated haemoglobin as $<6.5 \%, 6.5 \%$ to $<7.8 \%, 7.8 \%$ to $<9.1 \%$, and $\geq 9.1 \%$ (corresponding to $<48,48$ to $<62$, 62 to $<76$, and $\geq 76 \mathrm{mmol} / \mathrm{mol}$ ). We defined reference mothers as those who did not have a registered diagnosis of diabetes of any type (ie, type 1 or 2 , or gestational) any time before to 91 days after estimated conception (eTable 2). Baseline characteristics of mothers with type 1 diabetes included in the study cohort were similar to those of mothers with type 1 diabetes excluded from the study cohort because of no glycated haemoglobin measurement in the period around conception (eTable 3).

Gestational age was estimated through routine ultrasound examinations or, when such data were unavailable, calculated based on the date of the last menstrual period. Since 1990 in Sweden about 95\% of pregnant women have undergone ultrasonography in the second trimester to determine fetal age. ${ }^{25}$ We used data on gestational age to estimate the date of conception.

\section{Birth defects}

Our two main outcomes were major cardiac and non-cardiac birth defects recorded during the first year of life in the national patient register (primary or secondary diagnosis in inpatient and hospital based outpatient care) or the cause of death register (underlying or contributing causes). Outcome data were available to the end of 31 December 2015, allowing one year follow-up after birth for all included infants. We identified major birth defects using the International Classification of Diseases, 10th revision, and categorised in accordance with the European Surveillance of Congenital Malformations (EUROCAT) classification; we introduced minor modifications for this study (eTable 4). One infant could contribute to more than one category; if an infant had a major cardiac defect and a major non-cardiac defect, we counted the infant in both outcome categories. We also analysed any major birth defects as an additional outcome. In a post hoc analysis we also examined severe cardiac defects (see eTable 4 legend for definitions). Although a register based strategy to identify birth defects has not been validated in Swedish registers, validation of the Danish national patient register, which is comparable in content and structure to its Swedish counterpart owing to similarities in organisation of the registers and healthcare systems, reported that the overall positive predictive values for register based capture of cases were $90 \%$ for cardiac defects and $88 \%$ for birth defects overall. ${ }^{2627}$

\section{Statistical analysis}

Using generalised linear models with a robust sandwich estimator, we estimated risk ratios for birth defects, comparing infants of mothers with type 1 diabetes according to levels of glycated haemoglobin with infants of mothers without diabetes. To take into account possible dependence from repeated births from the same mother, we constructed models with maternal identity number as a cluster variable. Birth defects were assumed to follow a poisson distribution, and we estimated risk ratios using a log link function. Analyses were adjusted for calendar year of conception, maternal age, country of birth, living with a partner, education, parity, body mass index, smoking status, and other autoimmune diseases (eTable 5 lists definitions, data sources, and extent of missing values). For covariates with missing data, we used mode imputation if the extent of missingness was 
less than 5\%. For body mass index, which had 8.1\% missing values, we used a multiple imputation model with five iterations, including calendar year, maternal age, maternal height, country of birth, living with a partner, education, parity, smoking status, and other autoimmune diseases. The adjusted risk differencethat is, the adjusted absolute difference measured in number of cases with birth defects per 1000 infants comparing infants of mothers with type 1 diabetes according to levels of glycated haemoglobin with infants of mothers without diabetes, was calculated as (adjusted risk ratio-1)×the unadjusted risk among infants born to mothers without diabetes. We also used natural cubic spline regression, estimating the unadjusted number of cases with birth defects per 1000 infants according to glycated haemoglobin levels on a continuous scale (with knots at levels of $6 \%, 7 \%$, $8 \%, 9 \%$, and $10 \%$ ).

In an alternative analysis, we restricted the cohort to mothers with type 1 diabetes with glycated haemoglobin measurements in the 31-91 days after conception. This analysis was done to provide risk estimates associated with glycated haemoglobin measured in the clinical time window when pregnancy has been detected and women have their first antenatal visit. In additional analyses, we investigated associations between subgroups categorised according to alternative measures of poor glycaemic control and diabetes severity and the risk of birth defects, including hospital admission in the past year before conception because of diabetic acidosis; hospital admission in the year before conception because of diabetes; long term glycaemic control, defined as the mean of the last three glycated haemoglobin measurements; albuminuria; and estimated glomerular filtration rate (definitions in eTable 6).

We considered results to be statistically significant when the $95 \%$ confidence intervals did not include 1 for risk ratios and did not include 0 for risk differences. Data were analysed using SAS version 9.4.

\section{Patient involvement}

No patients or members of the public were involved in the development of research questions, the design of the study, or selecting our outcome measures. No patient was asked to advise on interpretation or writing up of results. We plan to disseminate the results of our research to the relevant patient community.

\section{Results \\ Cohort}

After exclusions, the cohort included 2458 infants of mothers with type 1 diabetes who had a glycated haemoglobin measurement within three months before or after estimated conception, and 1159865 infants of mothers without diabetes (fig 1). Table 1 shows the characteristics of the women. In both mothers with diabetes and mothers without diabetes the mean age at conception was 30 years, $7 \%$ were smokers, and $95 \%$ lived with a partner. Compared with mothers without diabetes, those with type 1 diabetes were more often overweight or obese, of Nordic origin, and more likely to have another autoimmune disease. With increasing levels of glycated haemoglobin, mothers with type 1 diabetes tended to be younger, be less likely to be living with a partner, have a lower education level, be overweight or obese, and more often be smokers.

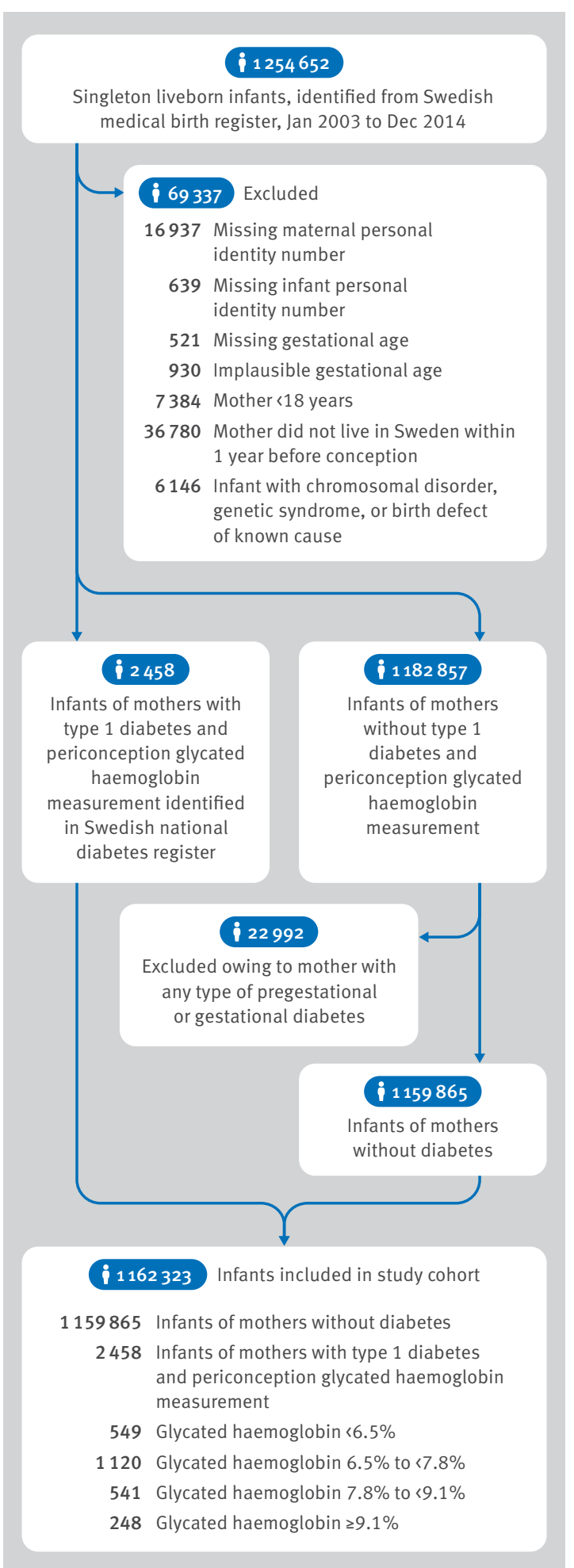

Fig 1 | Flow of participant inclusion in study cohort 


\begin{tabular}{|c|c|c|c|c|c|c|}
\hline \multirow[b]{2}{*}{ Characteristics } & \multirow{2}{*}{$\begin{array}{l}\text { No diabetes } \\
(\mathrm{n}=1159865)\end{array}$} & \multirow{2}{*}{$\begin{array}{l}\text { Type } 1 \text { diabetes } \\
\text { overall }(n=2458)\end{array}$} & \multicolumn{4}{|c|}{ Type 1 diabetes by glycated haemoglobin levels } \\
\hline & & & $<6.5 \%(n=549)$ & $6.5 \%$ to $<7.8 \%(n=1120)$ & $7.8 \%$ to $<9.1 \%(n=541)$ & $\geq 9.1 \%(n=248)$ \\
\hline Mean (SD) age (years) & $30.1(5.1)$ & $30.0(4.8)$ & $30.3(4.5)$ & $30.5(4.6)$ & $29.8(5.1)$ & $27.7(5.3)$ \\
\hline Nordic country of birth & $936370(80.7)$ & $2346(95.4)$ & $521(94.9)$ & $1086(97.0)$ & $506(93.5)$ & $233(94.0)$ \\
\hline Living with partner & $1097942(94.7)$ & $2343(95.3)$ & $535(97.4)$ & $1086(97.0)$ & $496(91.7)$ & $226(91.1)$ \\
\hline \multicolumn{7}{|l|}{ Year of conception: } \\
\hline $2002-04$ & $251087(21.6)$ & $312(12.7)$ & $68(12.4)$ & $136(12.1)$ & $76(14.0)$ & $32(12.9)$ \\
\hline 2005-09 & $482948(41.6)$ & $964(39.2)$ & $231(42.1)$ & $452(40.4)$ & $199(36.8)$ & $82(33.1)$ \\
\hline 2010-14 & $425830(36.7)$ & $1182(48.1)$ & $250(45.5)$ & $532(47.5)$ & $266(49.2)$ & $134(54.0)$ \\
\hline \multicolumn{7}{|l|}{ Duration of education (years): } \\
\hline$\leq 9$ & $92245(8.0)$ & $164(6.7)$ & $16(2.9)$ & $60(5.4)$ & $49(9.1)$ & $39(15.7)$ \\
\hline $10-12$ & $434594(37.5)$ & $1005(40.9)$ & $184(33.5)$ & $442(39.5)$ & $238(44.0)$ & $141(56.9)$ \\
\hline$\geq 13$ & $633026(54.6)$ & $1289(52.4)$ & $349(63.6)$ & $618(55.2)$ & $254(47.0)$ & $68(27.4)$ \\
\hline \multicolumn{7}{|l|}{ Parity: } \\
\hline 0 & $507497(43.8)$ & $1183(48.1)$ & $265(48.3)$ & $548(48.9)$ & $244(45.1)$ & $126(50.8)$ \\
\hline 1 & $433790(37.4)$ & $909(37.0)$ & $222(40.4)$ & $411(36.7)$ & $190(35.1)$ & $86(34.7)$ \\
\hline$\geq 2$ & $218578(18.8)$ & $366(14.9)$ & $62(11.3)$ & $161(14.4)$ & $107(19.8)$ & $36(14.5)$ \\
\hline \multicolumn{7}{|l|}{ Body mass index in early pregnancy: } \\
\hline$<18.5$ & $25061(2.2)$ & $10(0.4)$ & $1(0.2)$ & $2(0.2)$ & $5(0.9)$ & $2(0.8)$ \\
\hline 18.5 to $<25$ & $705835(60.9)$ & $1219(49.6)$ & $316(57.6)$ & $554(49.5)$ & $251(46.4)$ & $98(39.5)$ \\
\hline 25 to $<30$ & $304548(26.3)$ & $893(36.3)$ & $173(31.5)$ & $418(37.3)$ & $198(36.6)$ & $104(41.9)$ \\
\hline 30 to $<35$ & $88452(7.6)$ & $265(10.8)$ & $48(8.7)$ & $118(10.5)$ & $65(12.0)$ & $34(13.7)$ \\
\hline$\geq 35$ & $35969(3.1)$ & $71(2.9)$ & $11(2.0)$ & $28(2.5)$ & $22(4.1)$ & $10(4.0)$ \\
\hline Smoking in early pregnancy & $76079(6.6)$ & $164(6.7)$ & $18(3.3)$ & $46(4.1)$ & $45(8.3)$ & $55(22.2)$ \\
\hline Other autoimmune disease & $20171(1.7)$ & $259(10.5)$ & $53(9.7)$ & $113(10.1)$ & $63(11.6)$ & \\
\hline $\begin{array}{l}\text { Median (interquartile range) duration of } \\
\text { diabetes (years) }\end{array}$ & - & $16(9-22)$ & $13(4-20)$ & $17(10-23)$ & $17(11-22)$ & $16(10-20)$ \\
\hline $\begin{array}{l}\text { Median (interquartile range) glycated } \\
\text { haemoglobin level (\%) }\end{array}$ & - & $7.3(6.5-8.1)$ & $6.1(5.8-6.3)$ & $7.2(6.9-7.5)$ & $8.3(8.0-8.6)$ & $9.7(9.3-10.4)$ \\
\hline
\end{tabular}

\section{Main outcomes}

Major cardiac defects

In total, 122 cases of major cardiac defects were observed among 2458 infants of mothers with type 1 diabetes, including 18 cases among 549 infants whose mothers had a periconception glycated haemoglobin level of $<6.5 \%$, 55 cases among 1120 infants whose mothers had a periconception glycated haemoglobin level of $6.5 \%$ to $<7.8 \%, 24$ cases among 541 infants whose mothers had a periconception glycated haemoglobin level of $7.8 \%$ to $<9.1 \%$, and
25 cases among 248 infants whose mothers had a periconception glycated haemoglobin level of $\geq 9.1 \%$. Among 1159865 infants of mothers without diabetes, 17120 cases of major cardiac defects were observed. In analyses based on glycated haemoglobin levels, the adjusted risk ratios for major cardiac defects were 2.17 (95\% confidence interval 1.37 to 3.42 ) for $<6.5 \%, 3.17$ (2.45 to 4.11 ) for $6.5 \%$ to $<7.8 \%, 2.79$ (1.90 to 4.12$)$ for $7.8 \%$ to $<9.1 \%$, and $6.23(4.32$ to 9.00 ) for $\geq 9.1 \%$ versus infants of mothers without diabetes (table 2). The corresponding adjusted risk differences were 17

Table 2 | Association between type 1 diabetes in mothers and risk of major birth defects in offspring, according to glycated haemoglobin levels within three months before or after estimated conception

\begin{tabular}{|c|c|c|c|c|c|c|}
\hline \multirow[b]{2}{*}{ Variables } & \multirow[b]{2}{*}{ No diabetes } & \multirow{2}{*}{$\begin{array}{l}\text { Type } 1 \text { diabetes } \\
\text { overall }\end{array}$} & \multicolumn{4}{|c|}{ Type 1 diabetes by glycated haemoglobin levels } \\
\hline & & & $\ll 6.5 \%$ & $6.5 \%$ to $<7.8 \%$ & $7.8 \%$ to $<9.1 \%$ & $\geq 9.1 \%$ \\
\hline No of infants & 1159865 & 2458 & 549 & 1120 & 541 & 248 \\
\hline \multicolumn{7}{|l|}{ Major cardiac defects: } \\
\hline No of events & 17120 & 122 & 18 & 55 & 24 & 25 \\
\hline No of events per 1000 infants & 15 & 50 & 33 & 49 & 44 & 101 \\
\hline Unadjusted risk ratio $(95 \% \mathrm{Cl})$ & 1.00 (ref) & $3.37(2.83$ to 4.00$)$ & $2.23(1.42$ to 3.52$)$ & $3.33(2.58$ to 4.31$)$ & $2.99(2.03$ to 4.40$)$ & $6.82(4.72$ to 9.85$)$ \\
\hline Adjusted risk ratio $(95 \% \mathrm{Cl})^{*}$ & 1.00 (ref) & 3.19 (2.69 to 3.80$)$ & 2.17 (1.37 to 3.42$)$ & $3.17(2.45$ to 4.11$)$ & 2.79 (1.90 to 4.12$)$ & $6.23(4.32$ to 9.00$)$ \\
\hline $\begin{array}{l}\text { Unadjusted risk difference, } \\
\text { No of events }(95 \% \mathrm{Cl}) \text { per } 1000 \text { infants }\end{array}$ & - & $35(27$ to 44$)$ & 18 (6 to 37$)$ & $34(23$ to 49$)$ & 29 (15 to 50$)$ & $86(55$ to 131$)$ \\
\hline $\begin{array}{l}\text { Adjusted risk difference, } \\
\text { No of events }(95 \% \mathrm{Cl}) \text { per } 1000 \text { infants* }\end{array}$ & - & $32(25$ to 41$)$ & 17 (5 to 36$)$ & $32(21$ to 46$)$ & 26 (13 to 46) & 77 (49 to 118$)$ \\
\hline \multicolumn{7}{|l|}{ Major non-cardiac defects: } \\
\hline No of events & 20960 & 50 & 12 & 21 & 9 & 8 \\
\hline No of events per 1000 infants & 18 & 20 & 22 & 19 & 17 & 32 \\
\hline Unadjusted risk ratio $(95 \% \mathrm{Cl})$ & 1.00 (ref) & 1.13 (0.85 to 1.49$)$ & 1.21 (0.69 to 2.10$)$ & 1.04 (0.68 to 1.59$)$ & $0.92(0.48$ to 1.76$)$ & 1.79 (0.90 to 3.54$)$ \\
\hline Adjusted risk ratio $(95 \% \mathrm{Cl})^{\star}$ & 1.00 (ref) & 1.09 (0.82 to 1.44$)$ & 1.18 (0.68 to 2.07$)$ & 1.01 (0.66 to 1.54$)$ & 0.89 (0.46 to 1.69$)$ & 1.68 (0.85 to 3.33$)$ \\
\hline $\begin{array}{l}\text { Unadjusted risk difference, } \\
\text { No of events }(95 \% \mathrm{Cl}) \text { per } 1000 \text { infants }\end{array}$ & - & $2(-3$ to 9$)$ & $4(-6$ to 20$)$ & $1(-6$ to 11$)$ & $-1(-9$ to 14$)$ & $14(-2$ to 46$)$ \\
\hline $\begin{array}{l}\text { Adjusted risk difference, } \\
\text { No of events }(95 \% \mathrm{Cl}) \text { per } 1000 \text { infants* }\end{array}$ & - & $2(-3$ to 8$)$ & $3(-6$ to 19$)$ & $0(-6$ to 10$)$ & $-2(-10$ to 12$)$ & $12(-3$ to 42$)$ \\
\hline
\end{tabular}



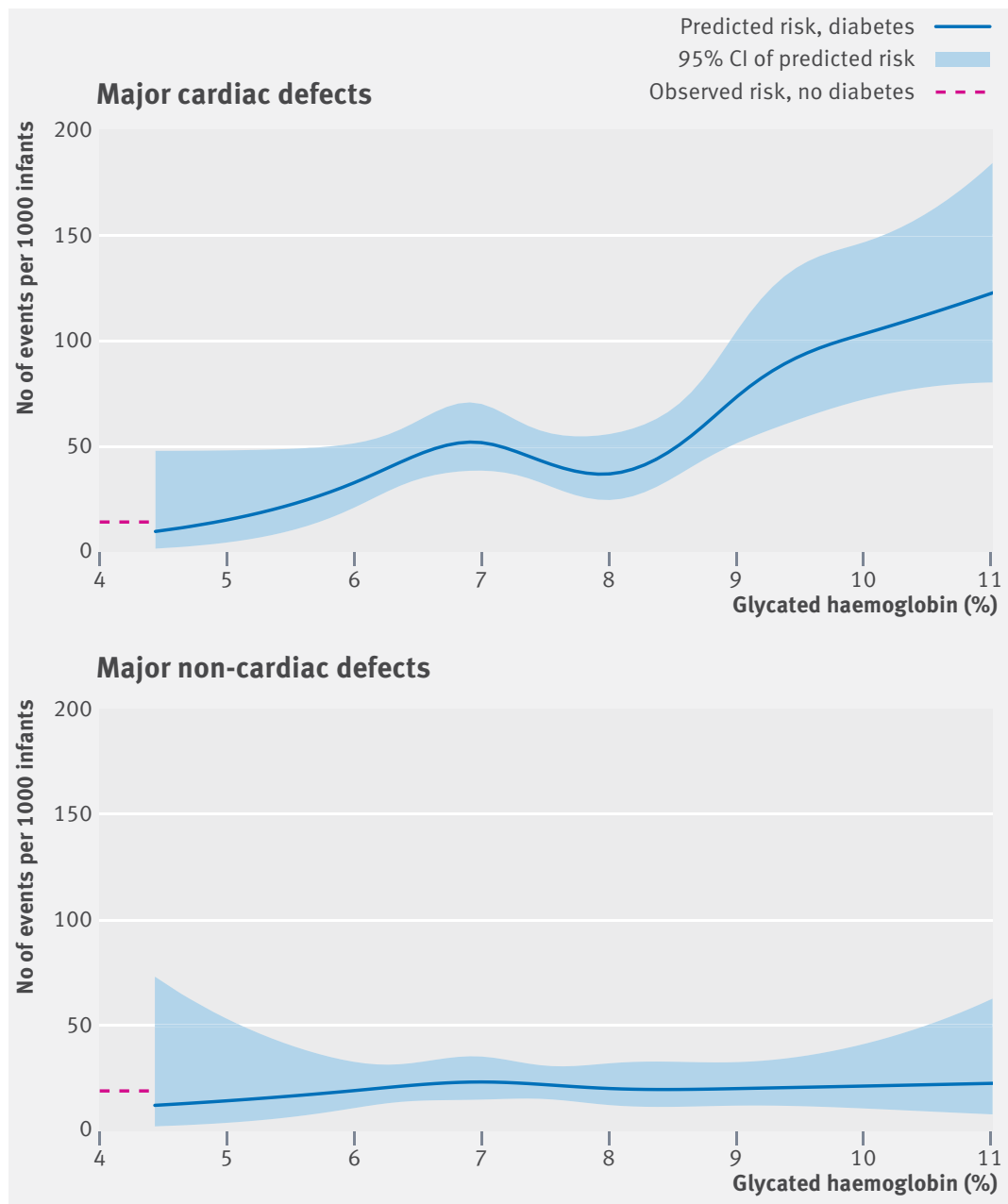

Fig 2 | Predicted unadjusted risk of major birth defects associated with type 1 diabetes according to maternal levels of glycated haemoglobin within three months before or after estimated conception

(95\% confidence interval 5 to 36), 32 (21 to 46), 26 (13 to 46), and 77 (49 to 118) cases per 1000 infants. Figure 2 shows the predicted unadjusted risk of major cardiac defects according to glycated haemoglobin levels on a continuous scale.

\section{Major non-cardiac defects}

In total, 50 cases of major non-cardiac defects were observed among 2458 infants of mothers with type 1 diabetes, including 12 cases among 549 infants whose mothers had a periconception glycated haemoglobin level of $<6.5 \%$, 21 cases among 1120 infants whose mothers had a periconception glycated haemoglobin level of $6.5 \%$ to $<7.8 \%$, nine cases among 541 infants whose mothers had a periconception glycated haemoglobin level of $7.8 \%$ to $<9.1 \%$, and eight cases among 248 infants whose mothers had a periconception glycated haemoglobin level of $\geq 9.1 \%$. Among 1159865 infants of mothers without diabetes, 20960 cases of major non-cardiac defects were observed. In analyses based on levels of glycated haemoglobin, the adjusted risk ratios for major noncardiac defects were 1.18 (95\% confidence interval 0.68 to 2.07 ) for $<6.5 \%, 1.01$ ( 0.66 to 1.54$)$ for $6.5 \%$ to $<7.8 \%, 0.89$ (0.46 to 1.69 ) for $7.8 \%$ to $<9.1 \%$, and
1.68 ( 0.85 to 3.33 ) for $\geq 9.1 \%$ versus infants of mothers without diabetes (table 2). Figure 2 shows the predicted unadjusted risk of non-cardiac defects according to glycated haemoglobin levels on a continuous scale.

\section{Additional analyses}

Supplementary eTable 7 shows results for any major birth defect (cardiac or non-cardiac), for which the adjusted risk ratios were 1.67 (1.18 to 2.37) for glycated haemoglobin levels $<6.5 \%, 2.05$ (1.65 to 2.55 ) for $6.5 \%$ to $<7.8 \%, 1.75$ (1.26 to 2.45 ) for $7.8 \%$ to $<9.1 \%$, and 3.63 (2.62 to 5.04 ) for $\geq 9.1 \%$.

When the cohort was restricted to 1068 mothers who had a glycated haemoglobin measurement registered 31-91 days after conception, a progressive increase in the risk of major cardiac defects with increasing glycated haemoglobin levels was found to be even more pronounced than in the main analysis: the adjusted risk ratios were 1.53 ( 0.73 to 3.17$)$ for a glycated haemoglobin level of $<6.5 \%, 3.78$ (2.66 to 5.37 ) for $6.5 \%$ to $<7.8 \%, 4.63$ (2.80 to 7.67 ) for $7.8 \%$ to $<9.1 \%$, and 8.43 (4.99 to 14.23 ) for $\geq 9.1 \%$ (eTable 8 ). The risk of major non-cardiac defects was not increased in any of the predefined glycated haemoglobin categories (eTable 8). 
Alternative measures of poor glycaemic control and severity of diabetes were also associated with an increased risk of major cardiac, but not non-cardiac, birth defects (fig 3). For instance, hospital admission for diabetic acidosis in the past year was associated with a 10-fold increased risk of major cardiac defects, whereas a 6.5 -fold increased risk of these defects was observed after hospital admission for any diabetes related condition. Similarly, both macroalbuminuria and an estimated glomerular filtration rate $<60 \mathrm{~mL} /$

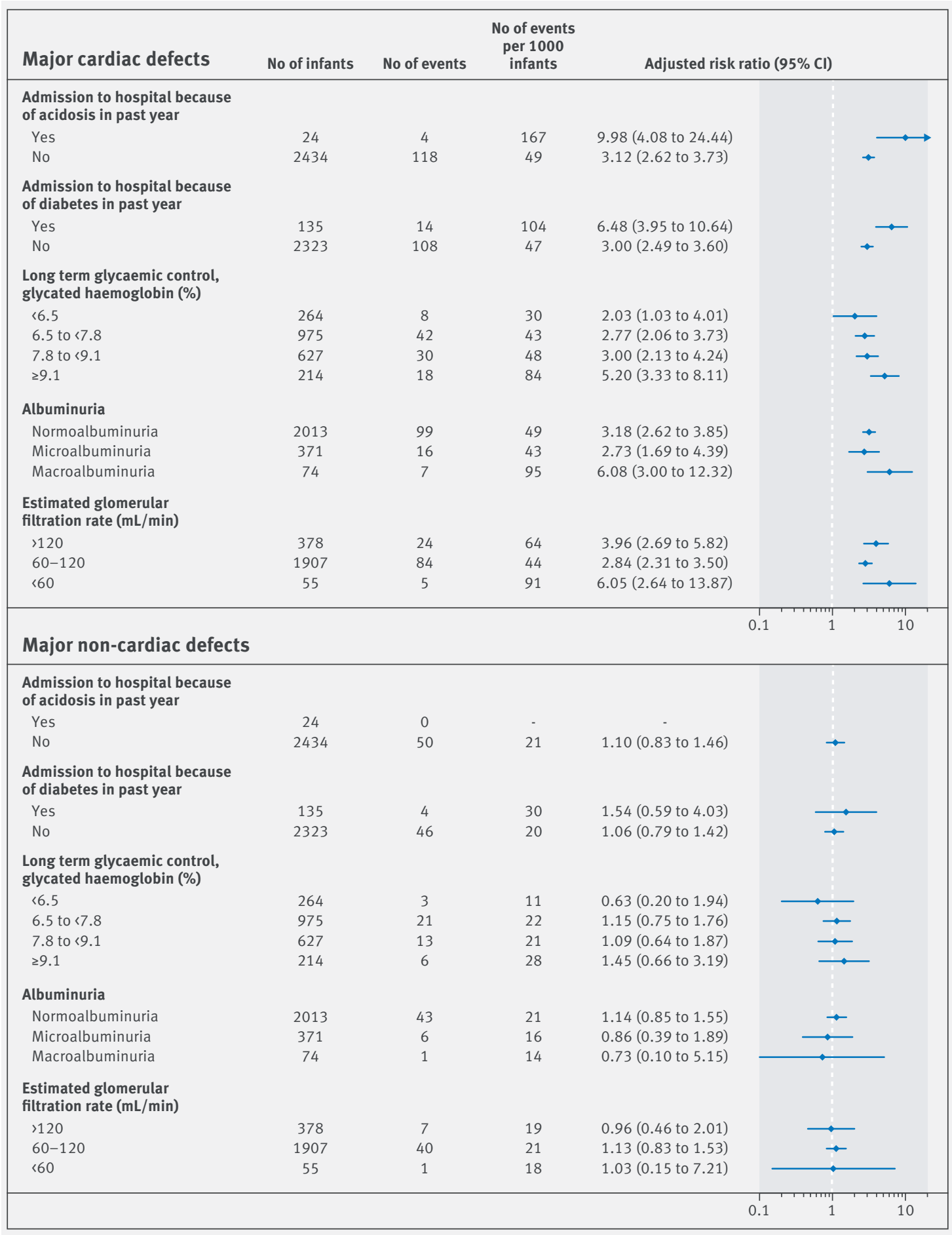

Fig 3 | Association between maternal type 1 diabetes and risk of major birth defects, using alternative measures of poor glycaemic control and diabetes severity. *Adjusted for calendar year, maternal age, country of birth, living with partner, education, parity, body mass index, smoking status, and other autoimmune diseases. The reference category in all analyses was infants born to mothers without diabetes (see table 2 for number of infants, number of events, and number of events per 1000 infants). Long term glycaemic control was defined as the mean of the last three measurements for glycated haemoglobin. The risk ratio plot uses a log scale 
min were each associated with more than a sixfold higher risk of major cardiac defects. Similar rates and adjusted risk ratios as in the main analyses were observed for long term glycaemic control, defined as the mean of the last three glycated haemoglobin measurements (fig 3).

In a post hoc analysis we identified 23 infants with a severe cardiac defect to mothers with type 1 diabetes (eTable 9 gives the absolute and relative risks according to glycated haemoglobin levels).

\section{Discussion}

In this population based study of 2458 liveborn singleton infants of mothers with type 1 diabetes, increasing glycated haemoglobin levels within the three months before or after estimated conception were associated with a progressively increased risk of major cardiac defects. The highest risk was observed in infants of mothers who had a glycated haemoglobin of $\geq 9.1 \%$, in whom the risk was increased more than sixfold, corresponding to 77 excess cases per 1000 infants. Even with glycated haemoglobin within target levels recommended by guidelines $(<6.5 \%),{ }^{15}$ -

17 the risk of major cardiac defects was more than twofold greater than in infants of mothers without diabetes. The risk of major non-cardiac defects was not statistically significantly increased at any of the four levels of glycated haemoglobin examined, although the study had limited statistical power for this outcome and was based on live births only.

\section{Comparison with other studies and interpretation of results}

Whereas type 1 diabetes is associated with an increased risk of birth defects (cardiac defects most consistently ${ }^{28}$ ), data on the impact of glycaemic control as measured by glycated haemoglobin level are limited. For instance, four papers ${ }^{10-13}$ underlie the American Diabetes Association's current recommendation of a target glycated haemoglobin level of $<6.5 \%$ ( $<48$ $\mathrm{mmol} / \mathrm{mol}$ ) in pregnancy. ${ }^{15}$ Two of these papers did not specifically investigate birth defects, ${ }^{11}{ }^{13}$ and the other two did not distinguish between cardiac and non-cardiac birth defects and had limited statistical power, precluding definitive conclusions about risks associated with the lowest glycated haemoglobin category. $^{10}{ }^{12}$ The most comprehensive study on the issue - a cohort study of 1367 infants from England ${ }^{29}$ found a linearly increasing risk of major birth defects with glycated haemoglobin levels $>6.3 \%$, but neither distinguished between cardiac and non-cardiac defects nor differentiated between type 1 and type 2 diabetes. ${ }^{29}$ Our study confirms previous findings, providing robust evidence for an association between worse periconception glycaemic control in type 1 diabetes and a progressive increase in the risk of birth defects. It substantially expands on those findings by characterising the magnitude of the association for both major cardiac and major non-cardiac defects based on a representative nationwide cohort. The study shows that the association was driven by cardiac defects and also that women with type 1 diabetes who have a periconception glycated haemoglobin level within target levels recommended by guidelines were at a substantially increased risk of delivering infants with major cardiac defects.

One potential mechanism behind an increasing risk of cardiac defects with higher glycated haemoglobin levels is that hyperglycaemia induces the expression of genes the products of which may influence the development of the heart in the fetus ${ }^{30}$; hyperglycaemia may also work through oxidative stress leading to mitochondrial dysfunction and apoptosis, thereby leading to cardiac defects. ${ }^{31}$

\section{Strengths and limitations of this study}

This study has several strengths. Firstly, the use of linked nationwide registers permitted the creation of a cohort with detailed characterisation of diabetes, including data on glycated haemoglobin levels. Although our study is a historical cohort study, we took advantage of prospectively collected data. Secondly, the design permitted comprehensive assessment of pregnancy characteristics and potential confounders. In this context, although increasing body mass index has been linked to increased risk of birth defects, ${ }^{32}$ we took body mass index into account in the current study through multivariable adjustment. Thirdly, the population based design increased the generalisability of the results. Fourthly, the large size of the study population provided statistical power to estimate risks across categories of glycated haemoglobin while distinguishing between cardiac and non-cardiac defects. Fifthly, data from the Swedish national diabetes register have been extensively validated and show high accuracy. ${ }^{20}$ Sixthly, the use of a standardised classification system for major birth defects is likely to have increased the specificity and external validity of the study. To increase sensitivity, we ascertained birth defects from several sources. Such an approach generated background rates in the general population as well as relative risks associated with diabetes overall that were similar to those reported in other Scandinavian countries. ${ }^{2833}$

This study has limitations. Data on glycated haemoglobin are entered into the national diabetes register once each year in association with routine visits, and our inclusion criteria for entry to the study cohort were based on those pregnant women with type 1 diabetes who had had their glycated haemoglobin measured in the period around conception. Because the timing of routine visits can be expected to be randomly distributed throughout the year, selection bias is unlikely. Indeed, baseline characteristics of the 1119 mothers with type 1 diabetes excluded because of no glycated haemoglobin value in the periconception period were similar to those of the included mothers.

We defined periconception glycated haemoglobin as a measurement performed within 90 days before to 91 days after conception. This was a pragmatic definition to capture glycaemic status around conception; however, because this definition extends 
for a period before pregnancy, it may not necessarily reflect glycaemic control in the first eight weeks of gestation, when blastogenesis and organogenesis take place. ${ }^{34}$ To address this and to provide risk estimates associated with glycated haemoglobin measured in the time window of the first antenatal visit, we carried out an alternative analysis based on women who had a glycated haemoglobin measurement registered 3191 days after conception. Results from this analysis were largely in line with the main analyses, although the progressive increase in the risk of cardiac defects appeared even more pronounced.

If diabetes leads to birth defects that more often result in induced or spontaneous abortion or stillbirth, risk estimates in a cohort based on live births, such as ours, may be biased towards the null. Given that diabetes has been reported to be associated with certain groups of non-cardiac defects, ${ }^{29} 35$ the absence of an association with non-cardiac defects in our study may have resulted from such bias. For instance, in Sweden, a high proportion of pregnancies with neural tube defects (which have been reported to be associated with diabetes) are terminated by induced abortion. Therefore, our results should not be taken to conclude that there is no association between type 1 diabetes and non-cardiac defects in general, but rather that there does not seem to be a strong association overall, or according to levels of glycated haemoglobin, specifically among liveborn infants. This is especially important considering the small number of cases of non-cardiac defects in our study. It should also be noted that outcome data were collected allowing for a one year follow-up after birth; birth defects that were first diagnosed in infants after the age of 1 year would not have been captured.

It is also possible that the previously demonstrated association between type 1 diabetes and birth defects has resulted in increased clinical surveillance for defects among infants of mothers with diabetes. Although such surveillance would result in bias towards increased risk, surveillance bias is unlikely to influence the relative difference between glycated haemoglobin categories, and, if present, would also have led to an association with non-cardiac defects. Finally, Swedish health registers do not record data on ethnicity or folic acid intake and hence we could not account for these factors.

\section{Conclusions and implications for clinical practice}

In this large nationwide cohort of liveborn infants of mothers with type 1 diabetes, increasingly worse glycaemic control within three months before or after estimated conception was associated with a progressively increased risk of major cardiac defects. Even with glycated haemoglobin within target levels recommended by guidelines $(<6.5 \%)$, the risk of major cardiac defects was more than twofold greater than in infants of mothers without diabetes. The risk of major non-cardiac defects was not statistically significantly increased at any of the four levels of glycated haemoglobin examined. Expanding on the evidence base for recommendations and guidelines about the clinical care of pregnant women with type 1 diabetes, these findings may indicate that yet lower target glycated haemoglobin levels than are currently recommended might be advisable. However, before any changes to practice guidelines are implemented, the totality of evidence on other adverse pregnancy, fetal, and infant outcomes in relation to poor glycaemic control-in particular the specific glycated haemoglobin levels at which these events occur-need to be carefully considered and weighed against the risks associated with intensified insulin treatment, such as hypoglycaemia.

\section{AUTHOR AFFILIATIONS}

${ }^{1}$ Department of Medical Epidemiology and Biostatistics, Karolinska Institutet, Stockholm, Sweden

${ }^{2}$ Department of Pediatrics, Örebro University Hospital, Sweden

${ }^{3}$ Division of Epidemiology and Public Health, School of Medicine, University of Nottingham, City Hospital, Nottingham, UK

${ }^{4}$ Department of Medicine, Columbia University College of Physicians and Surgeons, New York, NY, USA

${ }^{5}$ Clinical Epidemiology Division, Department of Medicine Solna, Karolinska Institutet, Sweden

${ }^{6}$ The National Diabetes Register, Centre of Registers Västra Götaland, Sweden

${ }^{7}$ Sahlgrenska Academy, Institute of Medicine, Department of Molecular and Clinical Medicine, University of Gothenburg, Gothenburg, Sweden

${ }^{8}$ Department of Women's and Children's Health, Karolinska Institutet, Stockholm, Sweden

${ }^{9}$ Department of Epidemiology Research, Statens Serum Institut, Copenhagen, Denmark

Contributors: JFL had full access to all of the data in the study and takes responsibility for the integrity of the data and the accuracy of the data analysis. He is the guarantor. All authors conceived and designed the study. All authors acquired, analysed, and interpreted the data. JFL drafted the manuscript. MN, JS, SG, A-MS, SF, OS, and BP critically revised the manuscript for important intellectual content. IS did the statistical analysis. BP obtained funding. JFL and BP supervised the study.

Funding: This project was supported by a grant from the Swedish Diabetes Foundation. BP was supported by an investigator grant from the Strategic Research Area Epidemiology Program at Karolinska Institutet and the Swedish Research Council (2016-01974). OS was supported by grants from the Swedish Research Council (20132429), by grants provided by the Stockholm County Council (ALF project 20130156), and by the strategic Research Program in Epidemiology at Karolinska Institutet. MN was supported by a grant from the Swedish Research Council (2013-3770). The funders had no role in in the design and conduct of the study; collection, management, analysis, and interpretation of the data; preparation, review, or approval of the manuscript; or the decision to submit the manuscript for publication.

Competing interests: All authors have completed the ICMJE uniform disclosure form at www.icmje.org/coi_disclosure.pdf and declare: no support from any organisation for the submitted work; however, BP has received an unrestricted research grant from the Novo Nordisk Foundation for a diabetes project not related to the present work. MN has received grants from Pfizer and Astra Zeneca for rheumatology projects unrelated to the present work and serves on the scientific advisory board for Itrim (compensation $<\$ 5000$ per year). None of the other authors have any conflicts of interest.

Ethical approval: This study was approved by the regional ethics committee in Stockholm (2016/1969-31-31).

Data sharing: No additional data available.

Transparency: The lead author (JFL) affirms that this manuscript is an honest, accurate, and transparent account of the study being reported; that no important aspects of the study have been omitted; and that any discrepancies from the study as planned (and, if relevant, registered) have been explained. 
This is an Open Access article distributed in accordance with the Creative Commons Attribution Non Commercial (CC BY-NC 4.0) license, which permits others to distribute, remix, adapt, build upon this work non-commercially, and license their derivative works on different terms, provided the original work is properly cited and the use is noncommercial. See: http://creativecommons.org/licenses/by-nc/4.0/

1 Weinstock RS, Xing D, Maahs DM, et al, T1D Exchange Clinic Network. Severe hypoglycemia and diabetic ketoacidosis in adults with type 1 diabetes: results from the T1D Exchange clinic registry. J Clin Endocrinol Metab 2013;98:3411-9. doi:10.1210/jc.2013-1589.

2 Rawshani A, Rawshani A, Franzén S, et al. Mortality and Cardiovascular Disease in Type 1 and Type 2 Diabetes. N Engl J Med 2017;376:1407-18. doi:10.1056/NEJMoa1608664.

3 Lind M, Svensson AM, Kosiborod M, et al. Glycemic control and excess mortality in type 1 diabetes. N Engl J Med 2014;371:197282. doi:10.1056/NEJMoa1408214.

4 Inkster ME, Fahey TP, Donnan PT, Leese GP, Mires GJ, Murphy DJ. Poor glycated haemoglobin control and adverse pregnancy outcomes in type 1 and type 2 diabetes mellitus: systematic review of observational studies. BMC Pregnancy Childbirth 2006;6:30. doi:10.1186/1471-2393-6-30.

5 Zhao E, Zhang Y, Zeng X, Liu B. Association between maternal diabetes mellitus and the risk of congenital malformations: A meta-analysis of cohort studies. Drug Discov Ther 2015;9:274-81. doi:10.5582/ddt.2015.01044.

6 Hoang TT, Marengo LK, Mitchell LE, Canfield MA, Agopian AJ. Original Findings and Updated Meta-Analysis for the Association Between Maternal Diabetes and Risk for Congenital Heart Disease Phenotypes. Am J Epidemiol 2017;186:118-28. doi:10.1093/aje/ kwx033.

7 Miller E, Hare JW, Cloherty JP, et al. Elevated maternal hemoglobin A1C in early pregnancy and major congenital anomalies in infants of diabetic mothers. N Engl J Med 1981;304:1331-4. doi:10.1056/ NEJM198105283042204.

8 Kitzmiller JL, Gavin LA, Gin GD, Jovanovic-Peterson L, Main EK, Zigrang WD. Preconception care of diabetes. Glycemic control prevents congenital anomalies. JAMA 1991;265:731-6. doi:10.1001/ jama.1991.03460060063025

9 Gabbay-Benziv R, Reece EA, Wang F, Yang P. Birth defects in pregestational diabetes: Defect range, glycemic threshold and pathogenesis. World J Diabetes 2015;6:481-8. doi:10.4239/wjd. v6.i3.481.

10 Jensen DM, Korsholm L, Ovesen P, et al. Peri-conceptional A1C and risk of serious adverse pregnancy outcome in 933 women with type 1 diabetes. Diabetes Care 2009;32:1046-8. doi:10.2337/dc082061.

11 Nielsen GL, Møller M, Sørensen HT. HbA1c in early diabetic pregnancy and pregnancy outcomes: a Danish population-based cohort study of 573 pregnancies in women with type 1 diabetes. Diabetes Care 2006;29:2612-6. doi:10.2337/dc06-0914.

12 Suhonen L, Hiilesmaa V, Teramo K. Glycaemic control during early pregnancy and fetal malformations in women with type I diabetes mellitus. Diabetologia 2000;43:79-82. doi:10.1007/ s001250050010.

13 Maresh MJ, Holmes VA, Patterson CC, et al, Diabetes and Pre-eclampsia Intervention Trial Study Group. Glycemic targets in the second and third trimester of pregnancy for women with type 1 diabetes. Diabetes Care 2015;38:34-42. doi:10.2337/ dc14-1755

14 Mills JL, Knopp RH, Simpson JL, et al. Lack of relation of increased malformation rates in infants of diabetic mothers to glycemic control during organogenesis. N Engl J Med 1988;318:671-6. doi:10.1056/ NEJM198803173181104

15 American Diabetes Association. 13. Management of Diabetes in Pregnancy. Diabetes Care 2017;40(Suppl 1):S114-9. doi:10.2337/ dc17-S016.

16 NICE. Diabetes in pregnancy: management from preconception to the postnatal period (https://www.nice.org.uk/guidance/ng3), 2015.

17 Blumer I, Hadar E, Hadden DR, et al. Diabetes and pregnancy: an endocrine society clinical practice guideline. J Clin Endocrinol Metab 2013;98:4227-49. doi:10.1210/jc.2013-2465.
18 Murphy HR, Bell R, Cartwright C, et al. Improved pregnancy outcomes in women with type 1 and type 2 diabetes but substantial clinic-to-clinic variations: a prospective nationwide study. Diabetologia 2017;60:1668-77. doi:10.1007/s00125-017-43143.

19 Ludvigsson JF, Otterblad-Olausson P, Pettersson BU, Ekbom A The Swedish personal identity number: possibilities and pitfalls in healthcare and medical research. Eur J Epidemiol 2009;24:659-67. doi:10.1007/s10654-009-9350-y.

20 Eliasson B, Gudbjörnsdottir S. Diabetes care--improvement through measurement. Diabetes Res Clin Pract 2014;106(Suppl 2):S291-4. doi:10.1016/S0168-8227(14)70732-6.

21 SNBHW. The Swedish Medical Birth Register: a summary of content and quality. http://www.socialstyrelsen.se/Lists/Artikelkatalog/ Attachments/10655/2003-112-3_20031123.pdf. Stockholm, Sweden: Swedish National Board of Health and Welfare, 2003.

22 Ludvigsson JF, Andersson E, Ekbom A, et al. External review and validation of the Swedish national inpatient register. BMC Public Health 2011;11:450.

23 Brooke HL, Talbäck M, Hörnblad J, et al. The Swedish cause of death register. Eur J Epidemiol 2017;32:765-73. doi:10.1007/s10654017-0316-1.

24 Ludvigsson JF, Almqvist C, Bonamy AK, et al. Registers of the Swedish total population and their use in medical research. Eur J Epidemiol 2016;31:125-36. doi:10.1007/s10654-016-0117-y.

25 Høgberg U, Larsson N. Early dating by ultrasound and perinatal outcome. A cohort study. Acta Obstet Gynecol Scand 1997;76:90712. doi:10.3109/00016349709034900

26 Larsen H, Nielsen GL, Bendsen J, Flint C, Olsen J, Sørensen HT. Predictive value and completeness of the registration of congenital abnormalities in three Danish populationbased registries. Scand J Public Health 2003;31:12-6. doi:10.1080/14034940210134194

27 Agergaard P, Hebert A, Bjerre J, Sørensen KM, Olesen C, Ostergaard JR. Children diagnosed with congenital cardiac malformations at the national university departments of pediatric cardiology: positive predictive values of data in the Danish National Patient Registry. Clin Epidemiol 2011;3:61-6. doi:10.2147/CLEP.S15627.

28 Øyen N, Diaz LJ, Leirgul E, et al. Prepregnancy Diabetes and Offspring Risk of Congenital Heart Disease: A Nationwide Cohort Study. Circulation 2016;133:2243-53. doi:10.1161/ CIRCULATIONAHA.115.017465

29 Bell R, Glinianaia SV, Tennant PW, Bilous RW, Rankin J. Periconception hyperglycaemia and nephropathy are associated with risk of congenital anomaly in women with pre-existing diabetes: a population-based cohort study. Diabetologia 2012;55;936-47. doi:10.1007/s00125-012-2455-y.

30 Basu M, Zhu JY, LaHaye S, et al. Epigenetic mechanisms underlying maternal diabetes-associated risk of congenital heart disease. $\mathrm{JCl}$ Insight 2017;2:95085. doi:10.1172/jci.insight.95085.

31 Ornoy A, Reece EA, Pavlinkova G, Kappen C, Miller RK. Effect of maternal diabetes on the embryo, fetus, and children: congenital anomalies, genetic and epigenetic changes and developmental outcomes. Birth Defects Res C Embryo Today 2015;105:53-72. doi:10.1002/bdrc.21090.

32 Persson M, Cnattingius S, Villamor E, et al. Risk of major congenital malformations in relation to maternal overweight and obesity severity: cohort study of 1.2 million singletons. BMJ 2017;357:j2563. doi:10.1136/bmi.j2563.

33 Leirgul E, Brodwall K, Greve G, et al. Maternal Diabetes, Birth Weight, and Neonatal Risk of Congenital Heart Defects in Norway, 1994-2009. Obstet Gynecol 2016;128:1116-25. doi:10.1097/ AOG.0000000000001694.

34 Carlson BM. Human Embryology and Developmental Biology. Saunders, 2013

35 Garne E, Loane M, Dolk H, et al. Spectrum of congenital anomalies in pregnancies with pregestational diabetes. Birth Defects Res A Clin Mol Teratol 2012;94:134-40. doi:10.1002/bdra.22886.

Supplementary information: description of registers, and etables1-9 